\title{
AVALIAÇÃO DA FORÇA MUSCULAR RESPIRATÓRIA E SINTOMAS DE DISPNEIA EM GESTANTES NO SEGUNDO TRIMESTRE GESTACIONAL
}

Ana Paula Barroca Silva, Ana Julia da Silva Viana, Ana Paula Menegolo, Stephanie Aparecida Gonçalves Medina, Flávio Danilo Mungo Pissulin, Francis Lopes Pacagnelli, Gabriela Andrade Piemonte Lopes

Universidade do Oeste Paulista - UNOESTE, Curso de Fisioterapia, Presidente Prudente, SP. e-mail: gabriela@unoeste.br

\section{RESUMO}

O objetivo do estudo foi avaliar a força muscular respiratória e sintomas de dispneia em gestantes saudáveis no segundo trimestre gestacional. Trata-se de um estudo de caráter descritivo, onde foram avaliadas sete gestantes, encaminhadas ao projeto de extensão universitária de atuação fisioterapêutica em mulheres gestantes atendidas no SUS. Foi aplicada a ficha de avaliação inicial para a coleta de dados pessoais e específicos da gestação; foi usada a escala de Borg com base em algumas situações do cotidiano da gestante para a percepção subjetiva de esforço e o teste de manovacuometria. A pesquisa mostrou que as forças musculares inspiratórias e expiratórias encontram-se diminuídas no segundo trimestre gestacional, e que a situação que obteve a maior percepção subjetiva de esforço foi limpar a casa. Concluise que houve diminuição da força muscular respiratória e que pode ter associação com os sintomas de dispneia apresentados.

Palavras-chave: gestação, dispneia, testes de função respiratória, músculos respiratórios.

\section{EVALUATION OF RESPIRATORY MUSCLE STRENGTH AND SYMPTOMS OF DYSPNEA IN THE SECOND GESTATIONAL TRIMESTER}

\begin{abstract}
The purpose of the study was to evaluate respiratory muscle strength and dyspnea symptoms in healthy pregnant women in the second trimester of pregnancy. This is a descriptive study, in which seven pregnant women were forwarded to the university extension project of physical therapy in pregnant women attended on SUS. An initial evaluation form was applied to collect personal and specific data related to gestation; the Borg scale based on some daily situations of the pregnant woman's life for the subjective perception of effort and the manovacuometry test. The research showed that inspiratory and expiratory muscle forces were decreased in the second gestational trimester, also the situation of greater subjective perception of effort was to clean the house. We concluded that there was a decrease in respiratory muscle strength and that it may be associated with dyspnea symptoms presented.

Keywords: pregnancy, dyspnea, respiratory function tests, respiratory muscles.
\end{abstract}

\section{INTRODUÇÃO}

A gestação é um evento natural e significativo na vida de uma mulher e está relacionada com alterações fisiológicas que são importantes para o desenvolvimento do feto e para adaptação do corpo da gestante durante todo o período gravídico'. Durante a gravidez existem diversas alterações importantes que ocorrem nos sistemas do corpo da mulher ${ }^{2}$. Entre essas alterações, ocorre o aumento no tamanho do útero, resultando na compressão do músculo 
diafragma e alterações metabólicas, que geram um aumento no consumo de oxigênio²

O músculo diafragma é o principal responsável pela respiração e esse aumento progressivo do volume abdominal causa impacto na função respiratória da gestante ${ }^{3}$. Já nas primeiras semanas, ocorre aumento no volume minuto devido ao aumento do volume corrente, e esta hiperventilação está diretamente relacionada com queixas de dispneia comumente apresentadas no período gestacional ${ }^{3}$. O ângulo subcostal e a circunferência torácica também aumentam $^{3}$. O déficit de força muscular e os sintomas de dispneia podem ser comuns durante o período gravídico, e são derivados das alterações histológicas que afetam a função respiratória e as trocas gasosas, ou também por complicações, podendo agravar o quadro para uma síndrome mais complexa de dispneia ${ }^{4}$.

As pressões musculares respiratórias podem ser avaliadas pela manovacuometria. Trata-se de um exame que mensura pressões negativas e positivas, de baixo custo e de fácil realização. Por meio dele, determina-se com precisão as alterações na musculatura respiratória, pois mensura a força inspiratória e expiratória, através da pressão inspiratória máxima (PImáx) e pressão expiratória máxima (PEmáx), respectivamente ${ }^{5}$.

A dispneia é relatada como o principal desconforto que a mulher vivencia durante 0 processo gestatório, ocorrendo em cerca de 60$70 \%$ das gestantes, e pode estar relacionada à maior sensibilidade do sistema respiratório causado pelo aumento da progesterona ${ }^{6}$, fazendo então com que as alterações que acometem o sistema respiratório sejam uma questão importante a ser estudada e compreendida, envolvendo a fisiologia e a mecânica ventilatória da mulher durante o período gestacional'. A escala de Borg classifica de forma subjetiva o esforço de uma pessoa a partir de sua própria percepção, sendo um conveniente instrumento para avaliar os sintomas de dispneia ${ }^{8}$.

Portanto, há uma necessidade de avaliar a força muscular respiratória durante a gestação, para que sejam obtidas informações importantes para o profissional que está em contato direto com a parturiente. Assim, as condutas clínicas e as orientações poderão ser feitas de uma maneira mais eficaz, juntamente com a necessidade da informação para as próprias gestantes, que cada vez mais procuram conhecimento sobre o momento em que estão vivendo.

Esse estudo teve como objetivo avaliar a força muscular respiratória e os sintomas de dispneia de gestantes saudáveis, no segundo trimestre gestacional.

\section{METODOLOGIA}

Esse estudo se caracteriza como descritivo e foi realizado numa clínica de Fisioterapia universitária, em Presidente Prudente, São Paulo, contando com gestantes encaminhadas participantes de um projeto de extensão de atuação fisioterapêutica em mulheres gestantes atendidas no SUS, no período de fevereiro a junho de 2018. O projeto foi submetido ao comitê de Ética em Pesquisa (CEP) da Unoeste e aprovado sob o protocolo CAAE 81350617.8.0000.5515, estando em concordância com a resolução 466/2012, CONEP. Participaram do estudo, gestantes saudáveis que foram instruídas em relação a todos os procedimentos e assinaram o termo de consentimento livre e esclarecido. Foram incluídas no estudo gestantes que se encontravam no segundo trimestre gestacional, acima de 18 anos, que não apresentassem patologias, e que não tivessem realizado atividade física nos três meses anteriores do dia da avaliação, e excluídas aquelas que não conseguissem executar o teste e o questionário corretamente, ou completamente, e aquelas que não estivessem hemodinamicamente estáveis durante a avaliação.

Primeiramente foi aplicada a avaliação para a coleta de dados pessoais e específicos da gestação. Posteriormente foi aplicada a Escala CR10 de Borg ${ }^{8}$ em relação às seguintes situações cotidianas: tomar banho, limpar a casa, lavar roupas e dirigir. A escala parte de zero, que corresponde a "absolutamente nada" e chega até dez, "extremamente forte", para percepção subjetiva do esforço. Em seguida foi realizado o teste de manovacuometria para mensuração das forças musculares respiratórias.

A medida da Pressão Inspiratória Máxima (PImáx), que reflete na força muscular inspiratória, foi feita com a paciente sentada, em $90^{\circ}$ e foi solicitado uma expiração até o volume residual (VR), depois solicitado para que realizasse uma inspiração máxima e mantivesse o esforço máximo por 1 a 2 seg. Para a mensuração da Pressão Expiratória Máxima (PEmáx), que mensura a força muscular expiratória, foi 
solicitado uma expiração máxima, e que fosse mantido o esforço por 1 a 2 seg. As manobras foram feitas com o manovacuômetro analógico Power Meter ${ }^{9}$. Este teste foi realizado em uma sala individualizada e foram dadas todas as orientações necessárias para a correta realização das avaliações. Os dados foram apresentados de maneira descritiva.

\section{RESULTADOS}

Todas as gestantes encaminhadas ao projeto de extensão foram avaliadas e inseridas no presente estudo, totalizando sete gestantes no segundo trimestre gestacional com média de idade de $28,28 \pm 8,67$ anos (Tabela 1 ).

Tabela 1. Caracterização das gestantes em segundo trimestre gestacional $(n=7)$.

\begin{tabular}{lc}
\hline Variáveis & Média \pm DP \\
\hline Idade (anos) & $28,28 \pm 8,67$ \\
Idade gestacional (semanas) & $19,42 \pm 4,61$ \\
Número de gestações & $1,57 \pm 1,13$ \\
\hline
\end{tabular}

Tabela 2. Força muscular respiratória das gestantes em segundo trimestre gestacional $(n=7$, média e DP).

\begin{tabular}{lcc}
\hline Variáveis & $\begin{array}{c}\text { Força musc. } \\
\text { respiratória }\end{array}$ & $\begin{array}{c}\text { Valor de } \\
\text { referência }\end{array}$ \\
\hline $\mathrm{PI}_{\text {máx }}\left(\mathrm{cmH}_{2} \mathrm{O}\right)$ & $64,57 \pm 21,88$ & $87,26 \pm 3,99$ \\
$\mathrm{PE}_{\text {máx }}\left(\mathrm{cmH}_{2} \mathrm{O}\right)$ & $74,28 \pm 25,07$ & $138,58 \pm 5,89$ \\
\hline
\end{tabular}

A Tabela 3 apresenta de forma descritiva, os valores da pressão inspiratória (PiMáx) e expiratória (PeMáx) medida, e prevista de cada gestante avaliada. Os valores indicam que $100 \%$ das gestantes avaliadas apresentaram redução das forças musculares respiratórias.

A percepção subjetiva de esforço no segundo trimestre gestacional, medida a partir da aplicação da Escala de Borg nas gestantes em situações cotidianas foi: tomar banho $(0,5 \pm 0,76)$, limpar a casa $(5 \pm 4,0)$, lavar roupas $(1,57 \pm 1,13)$ e dirigir $(0,14 \pm 0,37)$.

Os resultados da força muscular respiratória obtida no segundo trimestre gestacional estão apresentados na Tabela 2.

Tabela 3. Medidas de pressão inspiratória (PiMáx) e expiratória (PeMáx) medida e prevista das gestantes (G) em segundo trimestre gestacional.

\begin{tabular}{lccccccc}
\hline & $\mathbf{G 1}$ & $\mathbf{G 2}$ & $\mathbf{G 3}$ & $\mathbf{G 4}$ & $\mathbf{G 5}$ & $\mathbf{G 6}$ & $\mathbf{G 7}$ \\
\hline $\begin{array}{l}\mathbf{P i}_{\text {máx }} \text { medida } \\
\left(\mathrm{cmH}_{2} \mathrm{O}\right)\end{array}$ & 40 & 39 & 80 & 80 & 83 & 80 & 45 \\
$\mathbf{P i}_{\text {máx }}$ prevista & 83,45 & 94,03 & 90,81 & 80 & 85,29 & 84,83 & 83,91 \\
$\left(\mathrm{cmH}_{2} \mathrm{O}\right)$ & 40 & 60 & 80 & 80 & 120 & 80 & 60 \\
$\begin{array}{l}\mathrm{Pe}_{\text {máx }} \text { medida } \\
\left(\mathrm{cmH}_{2} \mathrm{O}\right)\end{array}$ & 123,95 & 148,59 & 143,83 & 140,43 & 135,67 & 134,94 & 133,63 \\
$\mathbf{P e}_{\text {máx }}$ prevista & $\left(\mathrm{cmH}_{2} \mathrm{O}\right)$ & & & & & & \\
\hline
\end{tabular}

\section{DISCUSSÃO}

Este estudo mostra em todas as gestantes avaliadas uma diminuição da força muscular respiratória no segundo trimestre gestacional, em relação aos parâmetros de normalidade para a idade delas, e sugere relação com os sintomas de dispneia apresentados, onde a situação de maior esforço foi limpar a casa.

Essa alteração pode estar diretamente relacionada com as alterações fisiológicas que a gestação promove no corpo da mulher. 0 crescimento do útero faz com que haja uma 
compressão do músculo diafragma, o principal responsável pela inspiração, onde sua posição de repouso se torna mais elevada ${ }^{10}$. Além disso, semanalmente, o diâmetro anteroposterior do tórax aumentará, fazendo com que os músculos abdominais sofram um alongamento, diminuindo sua tensão, visto que são essenciais para uma expiração eficaz. Essas alterações podem refletir na força muscular inspiratória e expiratória, respectivamente ${ }^{10}$.

Dados de um estudo que envolveu 150 gestantes na cidade de São Paulo, demonstram que a maior diferença com relação às pressões respiratórias entre os trimestres gestacionais ocorreram do primeiro para o segundo ${ }^{11}$, sendo o segundo trimestre o que chamaria mais atenção em relação às alterações e modificações respiratórias, podendo justificar a acentuada alteração encontrada neste estudo.

Já foi evidenciado que os valores encontrados na avaliação da força muscular respiratória máxima em gestantes, quando comparadas com as não gestantes encontram-se diminuídos ${ }^{7}$. Entretanto, alguns estudos nos mostram que as pressões respiratórias não se alteram significativamente durante as gestações ${ }^{12}$. As justificativas para essas diferenças podem ocorrer pela diferença de um equipamento para o outro, pelo calibre do aparelho, o fato da gestante realizar alguma atividade física, e colaboração da participante com o teste.

Além das alterações nos volumes e capacidades pulmonares, como a redução do volume de reserva expiratório e da capacidade residual funcional, acarretadas pela gestação, as alterações hormonais também afetam o trato respiratório ${ }^{13 .} \mathrm{O}$ progressivo aumento da progesterona e do estrogênio durante o período gestacional é um dos fatores que explicam a hiperventilação que a gestante apresenta, havendo então, um aumento da sensibidade do centro respiratório, o que pode apresentar relação direta com as queixas de dispneia existentes neste período ${ }^{14}$.

Portanto, a partir dessas prováveis alterações relacionadas à força muscular respiratória e os sintomas de dispneia, pode ser verificada a necessidade de atuações fisioterapeuticas desde o primeiro trimestre gestacional, para amenizar as queixas ao longo do período gestacional.

Destaca-se a conscientização do fortalecimento muscular respiratório no início da gestação no auxilio na prevenção de futuras condições relacionadas a queixas de dispneia, sendo uma das mais comuns apresentadas pelas mulheres gravídicas. Além do trabalho muscular respiratório, seria interessante terapêutica que incluísse a capacidade funcional dessa mulher, o que acarretaria melhorias na realização de suas atividades de vida diária.

Orientações quanto às alterações fisiológicas que acontecem neste período são fundamentais, bem como informações sobre como a fisioterapia pode intervir amenizando as consequências dessas alterações.

Importante citar também, a necessidade da realização de uma avaliação detalhada e individual da gestante, pois cada uma é única e com condições diferentes, podendo assim, ser realizada uma intervenção correta e de uma melhor forma, para garantir segurança e saúde para a mulher e para o feto.

As forças musculares inspiratórias e expiratórias apresentaram-se diminuidas em relação aos parâmetros de normalidade no segundo trimestre gestacional, sugere relação com os sintomas de dispneia apresentados, e a situação de maior esforço foi limpar a casa.

Atuar na prevenção pode ser uma conduta essencial para manter a funcionalidade dessa gestante, e oferecer uma melhor qualidade de vida neste momento único e especial, como a gestação. Dentre os diversos recursos disponíveis pelo fisioterapeuta no preparo pré-natal, os exercícios respiratórios adequados para este momento são considerados e recomendados principalmente para o alívio dos desconfortos.

\section{AGRADECIMENTOS E CONFLITO DE INTERESSE}

Agradecemos a professora Ana Paula Coelho Figueira Freire pelo suporte dado a metodologia do trabalho.

Os autores declaram não haver qualquer potencial conflito de interesse que possa interferir na imparcialidade deste trabalho científico.

\section{REFERÊNCIAS}

1. Constantine MM. Physiologic and pharmacokinetic changes in pregnancy. Front Pharmacol. 2014;5:65. DOI: https://doi.org/10.3389/fphar.2014.00065

2. Dubeau CE. The aging lower urinary tract. $J$ Urol. 2006;175;S11-S15.

DOI: https://doi.org/10.1016/S0022-5347(05)00311-3 
3. Lemos A, Souza Al, Andrade AD, Figueiroa JN, Cabral-Filho JE. Força muscular respiratória: comparação entre primigestas e nuligestas. J Bras Pneumol. 2011. 37(2):193-9. DOI: http://dx.doi.org/10.1590/S1809$\underline{29502011000300006}$

4. Lee SY, Chien DK, Huang $\mathrm{CH}$, Shih SC, Lee WC, Chang WH. Dyspnea in pregnancy. Taiwan J Obstet Gynecol. 2017 ;56(4):432-6. DOI: https://doi.org/10.1515/ipme.1975.3.3.154

5. Costa D, Gonçalves HA, Lima LP, Ike D, Cancelliero KM, Montebelo MIL. Novos valores de referência para pressões respiratórias máximas na população brasileira. J Bras Pneumol. 2010;36(3):306-12 DOI: http://dx.doi.org/10.1590/S1806$\underline{37132010000300007}$

6. Bonica J. Maternal respiratory changes during pregnancy and parturition. Clin Anest. 1974;10:1. DOI: http://dx.doi.org/10.1007/978-0-387-88602$\underline{21}$

7. Almeida LGD, Constâncio FL, Santos CVS. Análise comparativa das pe e pi máximas entre mulheres grávidas e não-grávidas e entre grávidas de diferentes períodos gestacionais. Rev Saúde Com. 2005;1(1):91-7.

8. Borg G. Escalas de Borg para a dor e o esforço percebido. São Paulo: Manole; 2000.

9. Caruso P, Albuquerque ALP, Santana PV, Cardenas LZ, Ferreira JG, et al. Métodos diagnósticos para avaliação da força muscular inspiratória e expiratória. J Bras Pneumol. 2015;41(2):110-23. DOI: http://dx.doi.org/10.1590/S1806$\underline{37132015000004474}$

10. Pinto AVA, Schleder C, Penteado C, GALLO RBS. Avaliação da mecânica respiratória em gestantes. Fisioter Pesq. 2015;22(4):348-54. DOI: http://dx.doi.org/10.590/1809$\underline{2950 / 13667922042015}$

11. Caromano F, Sayuri E, Cruz CMV, Candeloro JM, Burti JS, Andrade LZ. Mobilidade torácica e pressões respiratórias máximas durante a gestação. Fisioter Bras. 2006;7(1):5-7.
12. Silva EG, Godoy I, Antunes ICO, Silva EGS, Peraçoli JC. Respiratory parameters and exercise functional capacity in preeclampsia. Hypertens Pregnancy. 2010;29:301-9. DOI: https://doi.org/10.3109/10641950902779271

13. Koehler KF, Helguero LA, Haldosen LA, Warner M, Gustafssom JA. Reflections on the discovery and significance of estrogen receptor beta. Endocr Rev. 2005;26:475-8. DOI: https://doi.org/10.1210/er.2004-0027

14. LoMauro A, Aliverti A. Respiratory physiology of pregnancy: physiology masterclass. Breathe. 2015;11:297-301.

DOI: http://dx.doi.org/10.1183/20734735.008615 